\title{
Effects of lentivirus-mediated RNAi knockdown of NEDD9 on human lung adenocarcinoma cells in vitro and in vivo
}

\author{
JING-XIA CHANG ${ }^{1}$, FENG GAO ${ }^{1}$, GUO-QIANG ZHAO ${ }^{2}$ and GUO-JUN ZHANG ${ }^{1}$ \\ ${ }^{1}$ Department of Respiratory and Critical Care Medicine, The First Affiliated Hospital of Zhengzhou \\ University, Zhengzhou, Henan 450052; ${ }^{2}$ Department of Microorganisms and Immunization, \\ Preclinical Medicine of Zhengzhou University, Zhengzhou, Henan 450052, P.R. China
}

Received February 26, 2014; Accepted March 28, 2014

DOI: 10.3892/or.2014.3347

\begin{abstract}
The aim of the present study was to investigate the biological behavior of lung adenocarcinoma A549 cells following transfection with NEDD9-specific lentiviral particles in vitro and in vivo. NEDD9-specific lentiviral particles were chemically synthesized and transfected into the human lung adenocarcinoma A549 cell line. NEDD9 mRNA and protein levels were determined by fluorescence quantitative RT-PCR and western blotting. Cell proliferation was evaluated using soft agar colony formation assays and flow cytometric analysis. Migration and invasion were evaluated by wound-healing and Transwell assays and xenograft animal models. Transfection was successful, and expression levels of NEDD9 mRNA and protein in the lentivirus-NEDD9-siRNA group were downregulated. As indicated by soft agar colony formation assays, the number of clones in the siRNA group were significantly lower than the number of colonies in the blank and negative control groups $(\mathrm{P}<0.01)$. In addition, the percentage of cells in the $\mathrm{S}$ phase in the siRNA group was significantly lower than the percentages in the blank and negative control groups $(\mathrm{P}<0.05)$. Furthermore, as detected by cell migration and invasion assays, values of wound healing were increased and the number of invading cells were decreased in the siRNA group (both $\mathrm{P}<0.05$ ). We also showed that lentivirus-mediated NEDD9-siRNA decreased the growth potential of subcutaneous A549 xenografts in vivo. These data imply that knockdown of the NEDD9 gene results in suppression of tumor cell proliferation, migration, invasion and cell growth in vitro and in vivo. Lentivirus-mediated NEDD9siRNA may have potential therapeutic utility for human lung adenocarcinoma.
\end{abstract}

Correspondence to: Professor Guo-Jun Zhang, Department of Respiratory and Critical Care Medicine, The First Affiliated Hospital of Zhengzhou University, No. 1 Jianshe East Road, Erqit, Zhengzhou, Henan 450052, P.R. China

E-mail: guojunzhang666@yahoo.com

Key words: lung adenocarcinoma, NEDD9, lentivirus, proliferation, migration, invasion

\section{Introduction}

Lung cancer is the leading cause of cancer-related mortalityworldwide (1), and the 5-year survival rate is low. Non-small cell lung cancer (NSCLC) is subdivided into three subtypes: squamous cell carcinoma (SCC, $\sim 28 \%$ ), large-cell carcinoma (LCC, $\sim 24 \%$ ) and adenocarcinoma ( 48\%) (2). Most of the mortality associated with lung adenocarcinoma arises from uncontrolled metastases. Exploring the molecular mechanisms underlying lung adenocarcinoma metastasis is necessary to overcome lung adenocarcinoma metastasis and recurrence.

Scaffold protein neural precursor cell expressed, developmentally downregulated 9 (NEDD9), also known as HEF1 and Cas-L $(3,4)$, belongs to the family of Crk-associated substrate (CAS) proteins that regulate protein complexes controlling cell attachment, migration, invasion, cell cycle, apoptosis and oncogenic signal transduction (5-7). Overexpression of the NEDD9 protein is strongly linked to poor prognosis in cancer, as well as resistance to first-line chemotherapeutics in multiple tumor types including breast cancer (8), glioblastoma (9), melanoma (10) and gastrointestinal carcinoma $(11,12)$. High levels of NEDD9 mRNA and protein have been shown to be present in human lung carcinoma tissues, and are highly related to overall survival (OS) and to progression-free survival (PFS) (13-16).

Gene therapy for tumors has focused on gene replacement, antisense nucleic acid techniques, cytokine gene therapy and RNA interference (RNAi). RNAi is a post-transcriptional regulation method that provides a rapid means of depleting mRNA by introducing double-stranded RNA homologous to a particular message, causing its sequence-specific degradation. Using small interfering RNA (siRNA) to silence target genes is simple, specific and effective (17).

In the present study, NEDD9-specific lentiviral particles were chemically synthesized and transfected into the human lung adenocarcinoma A549 cell line. The inhibitory effect of siRNA on the expression of NEDD9 mRNA and protein was detected by RT-PCR and western blotting, and biological characteristics of A549 cells in vitro and in vivo including proliferation, cell cycle, migration and invasion were investigated. 


\section{Materials and methods}

NEDD9 siRNA lentivirus. siRNA targeting NEDD9 (GenBank Accession No. NM_182966) and a non-targeting RNA were chemically synthesized by GeneChem Technology Co., Ltd. (Shanghai, China). The target sequence was GTGTCCTAT TTCTTAGTGA, as determined by our previous research (18). The vehicle was GV118 (Fig. 1A). A negative control siRNA sequence (TTCTCCGAACGTGTCACGT) was used as a control for NEDD9 siRNA.

Lentiviral vector transduction. A549 cells were transduced with siRNA-expressing lentiviruses at a multiplicity of infection of 20 particles/cell in Dulbecco's modified Eagle's medium (DMEM) (HyClone, Logan, UT, USA) containing $5 \mu \mathrm{g} / \mathrm{ml}$ polybrene and incubated at $37^{\circ} \mathrm{C}$ in $5 \% \mathrm{CO}_{2}$ in 6-well plates. GFP expression was observed by fluorescence microscopy three days after transduction, and cells were harvested at 4 days after transduction for RT-PCR or western blot analysis.

RNA isolation and establishment of RT-PCR detection of $N E D D 9 m R N A$. Total RNA from three groups (blank control group, negative control group and siRNA group) was purified from cells using TRIzol reagent (Invitrogen, Carlsbad, CA, USA) according to the manufacturer's instructions. Firststrand cDNA was synthesized using $2.5 \mu \mathrm{g}$ total RNA and AMV retroviridase (Promega). Specific primers were designed for the mRNA sequence of the NEDD9 gene, and NEDD9 and GAPDH segments were amplified. Primers were from Shanghai Bioengineering Co. (Shanghai, China). NEDD9 gene primers were upstream 5'-CGTGGGTAAAAAGGTGTT CC-3' and downstream 5'-CAAGCCTCCAAACTCAGGAC-3' (amplified segment $124 \mathrm{bp}$ ); GAPDH primers were upstream 5'-TCGTGGAAGGACTCATGACC-3' and downstream 5'-AG GGATGATGTTCTGGAGAG-3' (amplified segment 97 bp).

NEDD9 mRNA was quantified by FQ-PCR using an ABI PRISM 7500 Sequence Detection System (Applied Biosystems, Foster City, CA, USA). Reactions consisted of $20 \mu \mathrm{l} 2 \mathrm{X}$ realtime PCR buffer, $0.5 \mu \mathrm{l}$ of upstream and downstream primers for NEDD9, $2 \mu \mathrm{l}$ reverse transcription product, $0.2 \mu \mathrm{l} \mathrm{Taq}$ DNA polymerase, and $\mathrm{ddH}_{2} \mathrm{O}$ to $40 \mu \mathrm{l}$. FQ-PCR reaction conditions consisted of $95^{\circ} \mathrm{C}$ for $3 \mathrm{~min} ; 95^{\circ} \mathrm{C}$ for $15 \mathrm{sec}, 65^{\circ} \mathrm{C}$ for $45 \mathrm{sec}$, for $40 \mathrm{cycles}$ and $72^{\circ} \mathrm{C}$ for $2 \mathrm{~min}$. GAPDH was simultaneously amplified as an internal reference. All reactions were performed in triplicate. Results were analyzed by calculating the $\mathrm{Ct}$ values for NEDD9 and GAPDH in samples, and the relative expression of NEDD9 mRNA in each group (the relative fold, RF) using the $2^{-\Delta \Delta C t}$ value was calculated (19).

Protein isolation and western blot analysis. NEDD9 protein expression was examined in A549 cells by western blot analysis. Cells were harvested in lysis buffer (2\% SDS, $50 \mathrm{mM}$ Tris, pH 7.4, $1 \mathrm{mM}$ EDTA, protease inhibitor mixture). The supernatant was collected after centrifugation and protein concentrations were determined using a BCA protein assay kit (Pierce, Rockford, IL, USA). Equal amounts of protein $(40 \mu \mathrm{g})$ were separated by $10 \%$ sodium dodecyl sulfatepolyacrylamide gel electrophoresis on $8 \%$ gels and transferred onto nitrocellulose membranes (HyClone). The membranes were blocked with $5 \%(\mathrm{v} / \mathrm{v})$ skimmed milk and probed with phospho-rabbit anti-human polyclonal primary antibodies for NEDD9 (93 kDa; Abcam, San Francisco, CA, USA). TIMP1 was detected using the rabbit monoclonal anti-human TIMP1 antibody (28.5 kDa; Santa Cruz Biotechnology, Santa Cruz, CA, USA). MMP9 was detected using a rabbit polyclonal antihuman MMP9 antibody (92 kDa; Santa Cruz Biotechnology) and cyclin $\mathrm{E}$ was detected using rabbit polyclonal anti-human cyclin E antibody ( $34 \mathrm{kDa}$; Santa Cruz Biotechnology). Incubations were carried out at $4^{\circ} \mathrm{C}$ overnight. Membranes were washed and incubated with horseradish peroxidaseconjugated goat anti-rabbit secondary antibody (Beijing Zhongshan Golden Bridge Biotechnology Co., Ltd., Beijing, China), at room temperature for $1 \mathrm{~h}$. Antibodies against $\beta$-actin (43 kDa; Santa Cruz Biotechnology) were used to measure protein loading. Bound antibodies were visualized using an electrochemiluminescence system (Amersham Pharmacia Biotech, Buckinghamshire, UK).

\section{Cell proliferation assay}

Soft agar colony formation assay. Growth medium (2XDMEM) was mixed 1:1 (v/v) with $1.2 \%$ low melting agar, and $300 \mu \mathrm{l}$ was plated in a layer in 6-well plates. Uninfected and infected cells were trypsinized, centrifuged, resuspended in $0.35 \%$ agar medium (equal volumes of $0.7 \%$ noble agar and culture medium), and plated onto the top agar at an initial concentration of 1,000 cells/well. Cells were fed every 3 days with $1.5 \mathrm{ml}$ growth medium. After culturing for 14 days, adherent cells were washed twice with PBS and fixed with 4\% paraformaldehyde for $30 \mathrm{~min}$ at room temperature. Colonies were visualized using cell staining with gentian violet (Beyotime Institute of Biotechnology Co., Ltd., Shanghai, China) and counted under a phase contrast microscopy with low magnification. All studies were performed at least three times.

Flow cytometric analysis. To determine the impact of overexpression of NEDD9 on cell cycle kinetics, A549 cells were collected at $72 \mathrm{~h}$ following transfection. Ribonuclease A (final concentration of $0.1 \mathrm{mg} / \mathrm{ml}$ ) was added to $1 \times 10^{6}$ cells, and the cells were incubated at $37^{\circ} \mathrm{C}$ for $30 \mathrm{~min}$, then on ice for $15 \mathrm{~min}$. A549 cells were resuspended in $50 \mu \mathrm{g} / \mathrm{ml}$ propidium iodide and incubated for $30 \mathrm{~min}$ in the dark at $4^{\circ} \mathrm{C}$. Cell cycle distribution and the percentage of cells with degraded DNA were determined by flow cytometric analysis (FCA) at an excitation wavelength of $488 \mathrm{~nm}$ using a FACSCalibur flow cytometer (Beckman Coulter, Brea, CA, USA) at the Medical and Science Research Institute of Zhengzhou University. Cell cycle histograms were obtained from three determinations, each with a total of 100,000 cells/treatment.

In vitro cell migration and invasion assays. To measure cell migration and invasion, wound-healing assays and Transwell assays were performed. For the wound-healing assays, cells $\left(5 \times 10^{5}\right.$ cells/well) were plated in 6 -well plates. After $12 \mathrm{~h}$, the confluent monolayer was scratched manually with a plastic $200-\mu 1$ pipette tip and after washing with PBS, the wounded monolayers of the cells were allowed to heal for $48 \mathrm{~h}$. Each migration assay was conducted at least three times independently.

Transwell cell migration assays were performed as previously described using Transwells $(8 \mu \mathrm{mol} / 1$ pore size 
polycarbonate membranes) (Corning, ChemiconA, USA). Cells from the various treatment groups $\left(1 \times 10^{5}\right)$ in $0.5 \mathrm{ml}$ serum-free medium were placed in the upper chamber. Lower chambers were loaded with $0.8 \mathrm{ml}$ medium containing $10 \%$ PBS. Cells that migrated into the lower chamber were counted after $24 \mathrm{~h}$ of incubation at $37^{\circ} \mathrm{C}$ in $5 \% \mathrm{CO}_{2}$. Non-migratory cells were removed. Invasive cells were stained with $0.2 \%$ crystal violet in $10 \%$ ethanol. To quantify the invasive cells, three independent fields per well were photographed under phase contrast microscopy. The number of cells per field were counted and averaged.

In vivo study of lung adenocarcinoma xenograft tumor models in nude mice. Thirty 4-week-old female BALB/c nude mice (weight 15-20 g) were purchased from the Beijing Lihua Experimental Animal Center (Beijing, China). Mice were housed in a temperature-controlled, pathogen-free animal facility with 12-h light and dark cycles. All animal experiments were performed under protocols approved by the Animal Center Animal Care and Use Committee of Zhengzhou University. After two weeks, the mice were divided into 3 groups ( $n=6$ per group): A549 control (blank), lentivirus negative-siRNA (negative) and lentivirus NEDD9-siRNA (siRNA) group. Cells $\left(5 \times 10^{6}\right.$ in $100 \mu \mathrm{l}$ PBS) with lentivirus expression vectors or untransfected cells were injected subcutaneously into the right flank of mice and cancer growth was observed. Tumor mass (xenograft) volume (20), calculated as volume $\left(\mathrm{mm}^{3}\right)=$ width $^{2}\left(\mathrm{~mm}^{2}\right) \times$ length $(\mathrm{mm}) / 2$ was detected every 2-3 days using a caliper. After 5 weeks, the mice were sacrificed and the tumors were harvested and weighed.

Statistical analysis. SPSS 17.0 (SPSS, Inc., Chicago, IL, USA) for Windows statistical software package was used for analysis. One-way analysis of variance (ANOVA) was used to investigate differences between the three groups. Values are presented as mean \pm standard deviation (SD). $\mathrm{P}<0.05$ indicates a significant difference, and $\mathrm{P}<0.01$ indicates a highly significant difference.

\section{Results}

NEDD9 siRNA synthesis. Recombinant plasmids were purified, confirmed by sequencing (Fig. 1B), packed into lentiviruses and named NEDD9/GV118-RNAi and LV-scrRNAi. Viruses were titered for use at dilutions of $\sim 1 \times 10^{8}$ titres $\mathrm{U} / \mathrm{ml}$ (data not shown).

Results of the transfection. A549 cells were transfected with lentiviruses containing siRNA directed against NEDD9 or a non-targeting negative control shRNA (Scr-siRNA). Cells showing green fluorescence were considered to be successfully transfected. As shown in Fig. 1C, the lentiviral infection rate was high.

Effects of lentivirus-mediated NEDD9 RNAi on expression of NEDD9 mRNA. Results of the RT-PCR (Fig. 1D) showed no significant difference in the levels of NEDD9 mRNA between the blank control and the negative control group $(0.89 \pm 6.05$-fold). The mRNA levels in the three siRNA groups $(0.21 \pm 5.42$-fold) were significantly lower than the mRNA levels in the blank control $(\mathrm{P}<0.05)$ and negative control groups $(\mathrm{P}<0.05)$. These data indicated that NEDD9 mRNA level in the A549 cells decreased significantly after transfection with NEDD9-siRNA. Transfection with NEDD9-specific siRNA resulted in degradation of NEDD9 mRNA to silence the NEDD9 gene.

Effects of lentivirus-mediated NEDD9 RNAi on NEDD9, TIMP1, MMP9 and cyclin E expression. Western blot analysis showed that levels of NEDD9, MMP9 and cyclin E protein in the siRNA group were significantly lower than levels in the blank and negative control groups, while the level of TIMP1 protein in the siRNA group was significantly higher than levels that in the blank and negative control groups $(\mathrm{P}<0.05$; Fig. $1 \mathrm{E})$. These data indicated that NEDD9-specific siRNA-silencing significantly reduced the levels of NEDD9, MMP9 and cyclin E protein and increased TIMP1 protein in the A549 cells.

Silencing of the levels of NEDD9 inhibits proliferation and alters A549 cell cycle. Soft agar colony formation assays were used to test the effects of NEDD9-siRNA on the proliferation of A549 cells in the three treatment groups. After culturing for 14 days, the NEDD9-siRNA group showed significantly fewer clones compared with the blank and negative control groups $(\mathrm{P}<0.01)$. No significant difference was found between the blank and negative control group ( $\mathrm{P}>0.05)$ (Fig. 2A).

Additionally, cell cycle analysis by FCM revealed that NEDD9-siRNA altered the cell cycle of A549 cells. The mean values are shown in Fig. 2B. No significant differences ( $P>0.05)$ were observed in the percentage of cells at each cell cycle phase between the blank and negative control group. The percentage of cells in the G0/G1 phase in the siRNA group $(68.0 \pm 2.1 \%)$ was significantly different $(\mathrm{P}<0.05)$ than that of the blank $(60.6 \pm 2.4 \%)$ and negative control groups $(62.0 \pm 1.4 \%)$. Similarly, a significant difference $(\mathrm{P}<0.05)$ was noted in the percentage of cells in the $\mathrm{S}$ phase in the siRNA group $(22.6 \pm 1.1 \%)$ vs. the blank $(30.5 \pm 1.7 \%)$ and negative control groups $(30.0 \pm 1.4 \%)$. However, no significant difference ( $P>0.05)$ in the percentage of cells in the $\mathrm{G} 2 / \mathrm{M}$ phase was observed in the siRNA group $(9.3 \pm 1.1 \%)$, relative to the blank $(8.8 \pm 1.6 \%)$ and negative control groups $(8.1 \pm 1.3 \%)$. Silencing of NEDD9 increased the percentage of cells at the G0/G1 phase and decreased the percentage of cells at the $S$ phase. These results suggest that siRNA treatment arrested cells at the G1/S checkpoint and delayed S phase.

Silencing of the NEDD9 gene suppresses A549 cell migration and invasion in vitro. A549 cells were seeded in 6-well plates and wounded the next day. Images were captured at $0 \mathrm{~h}$ and $48 \mathrm{~h}$ after wounding. Fig. $2 \mathrm{C}$ shows that cell migration was significantly decreased in the siRNA group when compared to that in the blank and negative control groups $(\mathrm{P}<0.05)$. Transwell cell migration assays showed significantly fewer invading cells in the siRNA group when compared to the number of invading cells in the blank and negative control groups (Fig. 2D; $\mathrm{P}<0.01$ ). The siRNA group had $68 \pm 10$ invading cells compared with $148 \pm 23$ in the blank control group and $136 \pm 20$ in the negative control group. These results demonstrated that transfection with NEDD9 siRNA reduced the migration and invasion of A549 cells. 
A

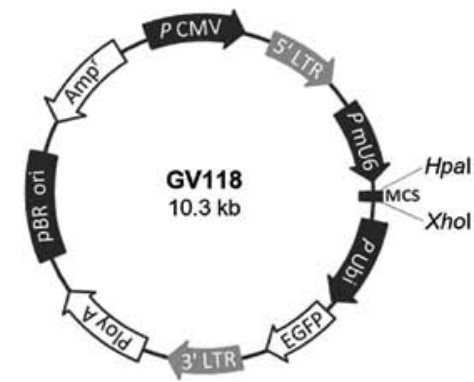

C

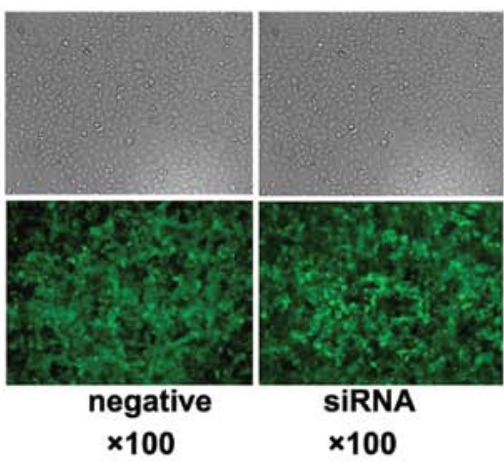

E1

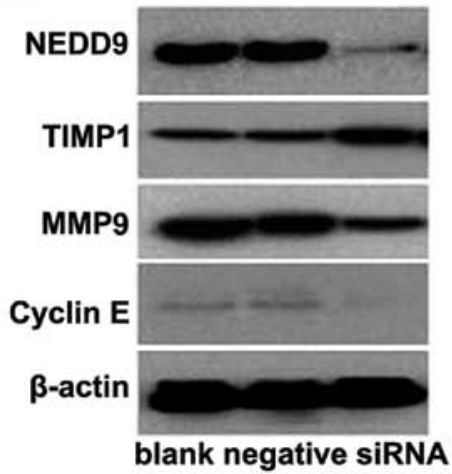

B

NEDD9/GV118-RNAi

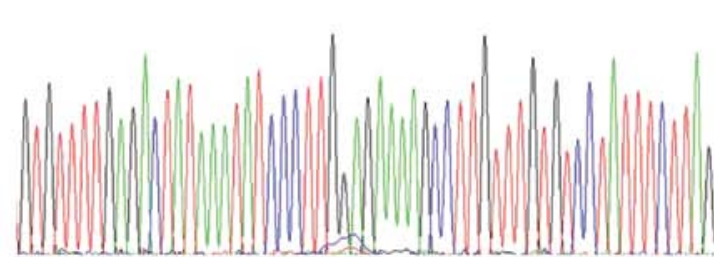

LV-ScrRNAi

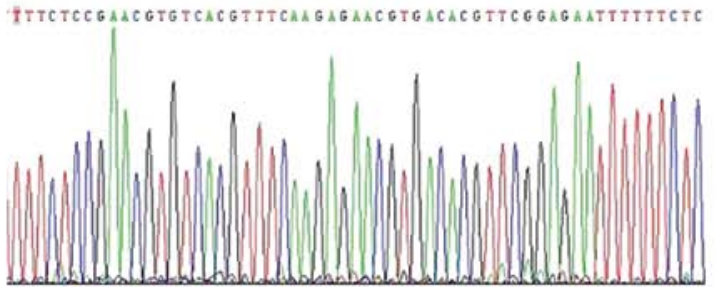

D

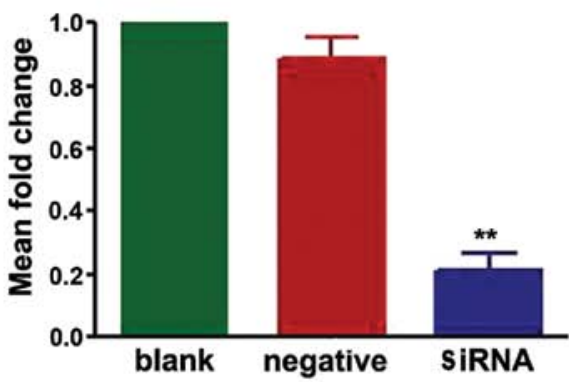

E2

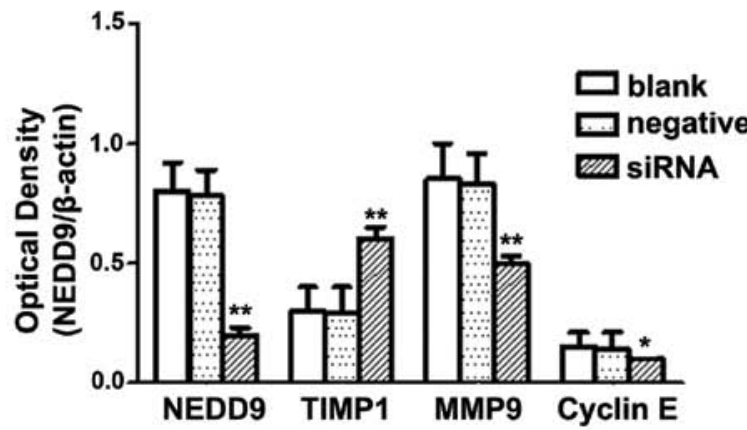

Figure 1. Construction and identification of lentivirus-mediated RNAi knockdown of NEDD9. (A) Schematic representation of lentivirus vehicle GV118. (B) NEDD9/GV118-RNAi and LV-scrRNAi were confirmed by sequencing. (C) Micrographs of green fluorescence cells transfected with LV-scrRNAi and NEDD9/GV118-siRNA (original magnification, x100). NEDD9-specific siRNA reduced NEDD9 mRNA levels in the A549 cells. (D) Histogram of NEDD9 mRNA levels. (E1) Western blot analysis of NEDD9, TIMP1, MMP9 and cyclin E in the three groups. (E2) Histogram of NEDD9, TIMP1, MMP9 and cyclin E protein levels. NEDD9-specific siRNA reduced NEDD9, MMP9 and cyclin E protein levels in the A549 cells, while TIMP1 protein increased. ${ }^{* *}<<0.01$, ${ }^{*} \mathrm{P}<0.05$ compared with the blank and negative control groups.

NEDD9/GV118-RNAi suppresses tumor growth in vivo. To determine whether knockdown of NEDD9 inhibits lung tumor growth in vivo, the growth of lung adenocarcinoma tumor xenografts in the three treatment groups was compared. Measurement of tumor volumes began once subcutaneous tumors became palpable and continued until tumors were excised on day 35. Then tumor tissues were harvested and weighed. The results showed that xenografts of the NEDD9siRNA group had a lower tumor volume $\left(100.71 \pm 42.73 \mathrm{~mm}^{3}\right)$ on day 35 than xenografts of the negative $\left(402.43 \pm 97.21 \mathrm{~mm}^{3}\right)$ $(\mathrm{P}<0.01)$ or blank groups $\left(340.92 \pm 66.06 \mathrm{~mm}^{3}\right) \quad(\mathrm{P}<0.01)$ (Fig. 3A-C). The average weight of tumors derived from cell of the NEDD9-siRNA group $(0.13 \pm 0.06 \mathrm{~g})$ was significantly reduced compared to the weights of tumors derived from cells from the negative $(0.35 \pm 0.10 \mathrm{~g})(\mathrm{P}<0.01)$ and blank control groups $(0.37 \pm 0.13 \mathrm{~g})(\mathrm{P}<0.01)$ (Fig. 3D).

\section{Discussion}

Human lung adenocarcinoma, a leading cause of cancerrelated mortality worldwide, exhibits features of invasiveness and undergoes early metastasis. Human lung adenocarcinoma is increasing in incidence and is a threat to human health. Understanding the pathological mechanisms of lung adenocarcinoma and identifying treatment targets are crucial.

CAS proteins mediate cell spreading $(21,22)$ and are important in driving cell migration $(23,24)$. NEDD9 is a member of the CAS protein family and an invasion-related 
A1

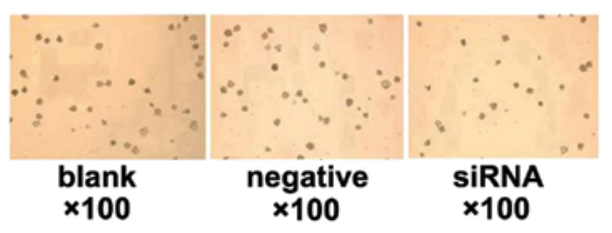

B1

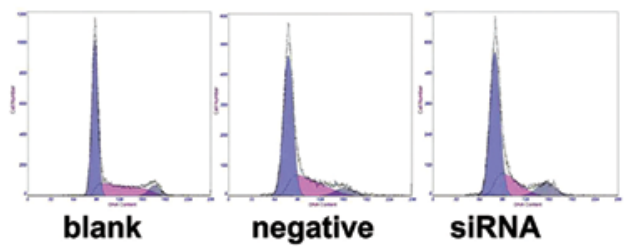

C1

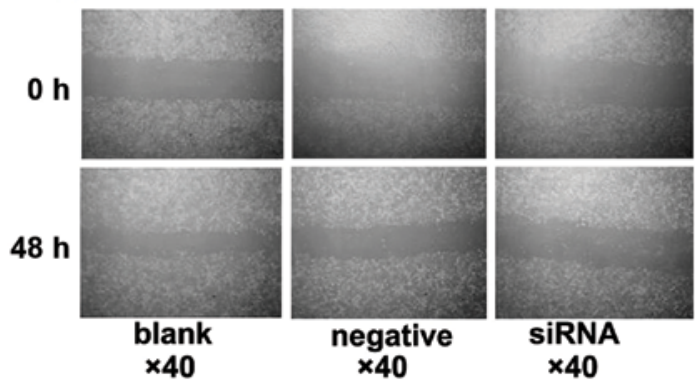

D1

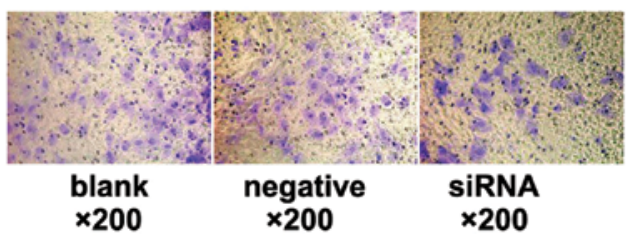

A2

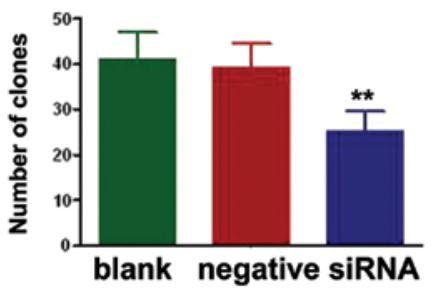

B2

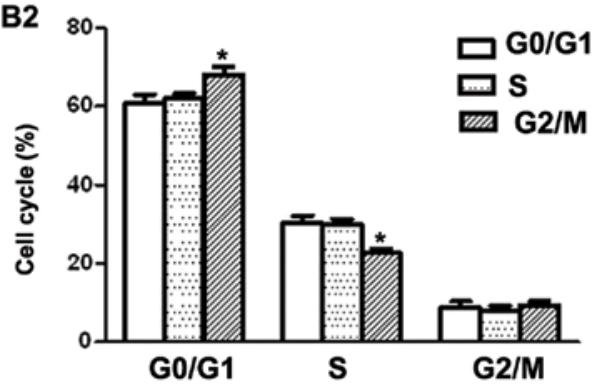

C2
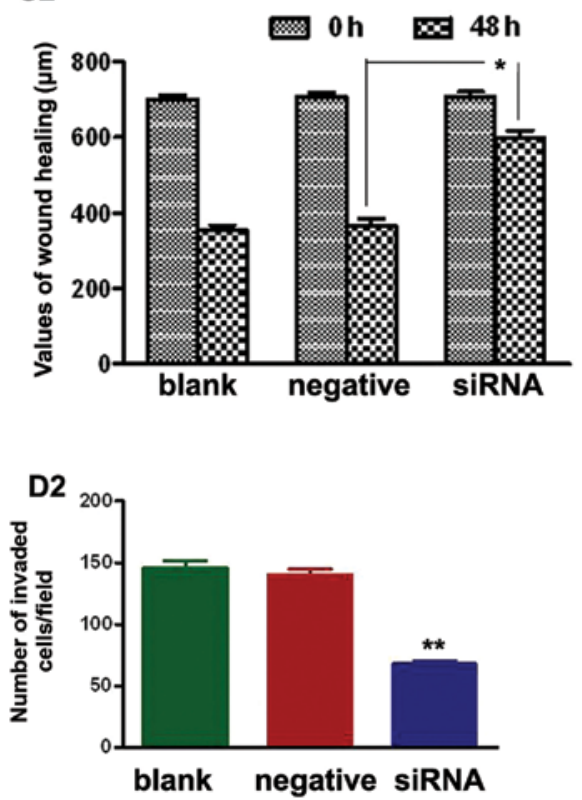

Figure 2. Knockdown of NEDD9 inhibits proliferation and suppresses cell migration and invasion in vitro. (A1) Colony formation in soft agar by A549 cells in the blank, negative control and siRNA groups. (A2) Histogram of the number of clones in the three treatment groups. (B1) DNA of A549 cells from the blank, negative control and siRNA groups by FCM. (B2) Histogram of the percentage of cells in cell cycle phases in the three treatment groups. (C1) A549 cells were scratched with a $200-\mu 1$ pipette tip. Representative images of wound healing were obtained at the time of the scratch and $48 \mathrm{~h}$ after. (C2) Histogram of the wound healing values for the three treatment groups. (D1) Cells in the blank, negative control and siRNA groups that transversed the Transwell. (D2) Histogram of A549 cells in the invasion assays. ${ }^{* *} \mathrm{P}<0.01,{ }^{*} \mathrm{P}<0.05$ compared with the blank and negative control groups.

and metastasis-related gene found in many tumor types (8-12). Our previous research showed that NEDD9 is overexpressed in lung adenocarcinoma tissues (13). Kondo et al (14) demonstrated that tyrosine phosphorylation of NEDD9 is reduced by inhibition of epidermal growth factor receptor protein (EGFR) in NSCLC cell lines. They suggested that NEDD9 is a promising biomarker for NSCLC prognosis, and its expression promotes NSCLC metastasis. Miao et al (16) showed that NEDD9 was overexpressed in 56.2\% (59/105) of NSCLC samples compared to normal lung tissues.

Although NEDD9 lacks known enzymatic function, it contains many functional modules for protein interaction, leading to its classification as a scaffolding protein. Since NEDD9 appears to lack catalytic activity, it is not immediately promising as a target for directed drug development, unless through agents intended to disrupt protein-protein interactions, or through an siRNA-based approach to deplete NEDD9 levels globally (7). siRNA-mediated knockdown of NEDD9 was found to reduce the number of cells undergoing mitosis, and lead to cleavage furrow regression and multinucleation $(24,25)$. In support of the development of NEDD9-directed drugs or siRNAs, genetic NEDD9 knockout animals have relatively limited defects, implying that loss of NEDD9 is well tolerated (26). RNA interference technology is an important tool for studying gene function (27), but siRNA-mediated gene silencing is maintained for only a short time (5-7 days) and usually transfection efficiency is low (28). Therefore, vectormediated RNA interference is used. Recently, lentiviral 
A

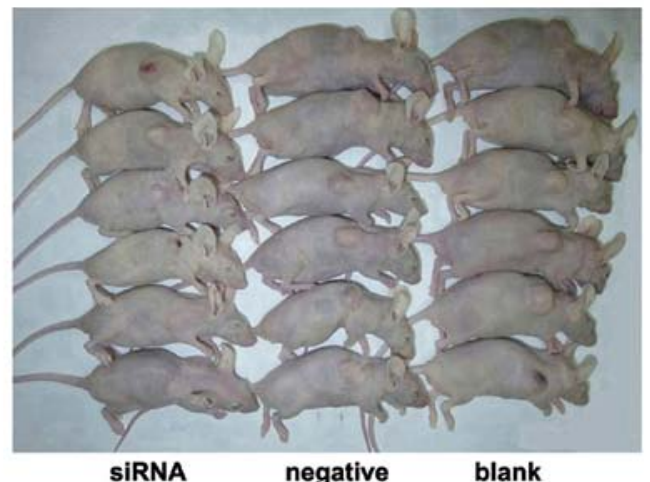

C

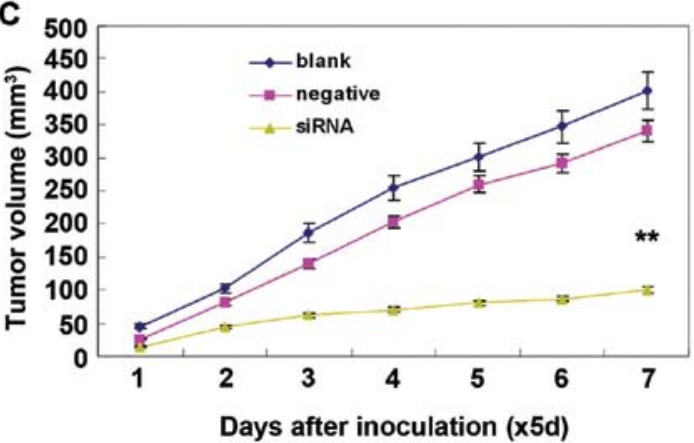

B

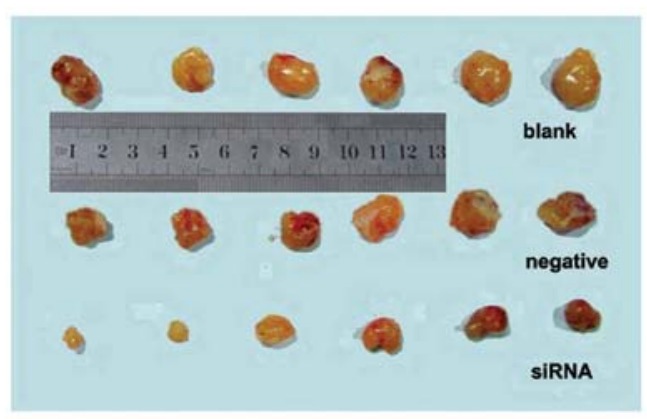

D

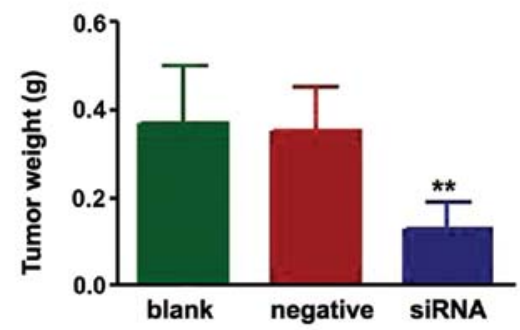

Figure 3. Lentivirus-mediated NEDD9-siRNA inhibits growth of tumor cells in vivo. (A and B) Xenograft mice and tumors at the end of the experiment on day 35. (C) Growth curves of human tumor xenografts for the three treatment groups. Data are means \pm SD. ${ }^{* *} \mathrm{P}<0.01$, compared with the blank and negative control groups. (D) Weights of dissected tumors. ${ }^{* *} \mathrm{P}<0.01$, compared with the blank and negative control groups.

technology has been developed, with advantages such as low immunogenicity, wide range of infection capabilities, and efficient integration into a host cell genome to express stable siRNA. Lentiviral-mediated RNA interference has become popular and can overcome the shortcomings of chemically synthesized siRNA (29).

In the present study, we hypothesized that downregulation of NEDD9 expression in the A549 cell line would affect lung adenocarcinoma tumorigenesis and tumor biological characteristics. To test this hypothesis, we used lentivirus-mediated siRNA silencing to suppress NEDD9 expression. RT-PCR and western blot analysis showed that NEDD9 mRNA and protein levels were both reduced in the cultured A549 lung adenocarcinoma cells after NEDD9 was silenced (Fig. 1C and 1D).

MMP9, cyclin E and TIMP1 are important for cell proliferation. We found that MMP9 and cyclin E were downregulated, but TIMP1 was increased in the A549 cells when NEDD9 was knocked down (Fig. 1E). This indicated that lentivirus-mediated RNAi knockdown of NEDD9 inhibited cell proliferation. Soft agar colony formation assays and FCM results showed that the colony formation number was decreased and the cell cycle was altered in the A549 cell line transfected with the lentivirus-NEDD9-siRNA (Fig. 2A and B). Migration and in vitro invasion were also suppressed (Fig. $2 \mathrm{C}$ and D). Our findings showed that lentivirus-NEDD9-siRNA transfection of lung adenocarcinoma cells markedly suppressed proliferation and migration and invasion ability. Tumorigenicity was also inhibited by lentivirus-mediated RNAi knockdown of NEDD9 expression in the A549 cell lines in vivo. Tumor growth was greatly reduced in the lentivirus-mediated NEDD9-siRNA transfected cell tumors while negative siRNA-transfected xenografts and control grafts grew aggressively in the mice (Fig. 3).

Taken together, our findings revealed that knockdown of NEDD9 resulted in inhibition of proliferation and cell cycle changes, and led to suppressed tumor cell migration and invasion. Lentivirus-NEDD9-siRNA effectively inhibited human lung adenocarcinoma cell growth in vivo and could have therapeutic utility for human lung adenocarcinoma. However, our results were based on a single cell line, and further research is needed to determine the differential expression of NEDD9 in different types of cells in vitro and in vivo.

\section{Acknowledgements}

The authors thank Dr P.J. Wang and his colleagues at the Research Centre for Molecular Oncology of Zhengzhou University. The present study was supported by a grant from the Science and Technology Agency of Henan (grant no. 122300410363).

\section{References}

1. Jemal A, Bray F, Center MM, et al: Global cancer statistics. CA Cancer J Clin 61: 69-90, 2011.

2. Tuveson DA and Jacks T: Modeling human lung cancer in mice: similarities and shortcomings. Oncogene 18: 5318-5324, 1999.

3. Law SF, Estojak J, Wang B, et al: Human enhancer of filamentation 1, a novel p130Cas-Like docking protein, associates with FAK, and induces pseudohyphal growth in Saccharomyces cerevisiae. Mol Cell Biol 16: 3327-3337, 1996.

4. Minegishi M, Tachibana K, Sato T, et al: Structure and function of Cas-L, a 105-kD Crk-associated substrate-related protein that is involved in $\beta-1$ integrin-mediated signaling in lymphocytes. J Exp Med 184: 1365-1375, 1996. 
5. Singh M, Cowell L, Seo S, et al: Molecular basis for HEF1/ NEDD9/Cas-L action as a multifunctional co-ordinator of invasion, apoptosis and cell cycle. Cell Biochem Biophys 48: $54-72,2007$

6. O'Neill GM, Seo S, Serebriiskii IG, et al: A new central scaffold for metastasis: parsing HEF1/Cas-L/NEDD9. Cancer Res 67: 8975-8979, 2007.

7. Tikhmyanova N, Little JL and Golemis EA: CAS proteins in normal and pathological cell growth control. Cell Mol Life Sci 67: 1025-1048, 2010

8. Minn AJ, Gupta GP, Siegel PM, et al: Genes that mediate breast cancer metastasis to lung. Nature 436: 518-524, 2005.

9. Natarajan M, Stewart JE, Golemis EA, et al: HEF1 is a necessary and specific downstream effector of FAK that promotes the migration of glioblastoma cells. Oncogene 25: 1721-1732, 2006.

10. Kim M, Gans JD, Nogueira C, et al: Comparative oncogenomics identifies NEDD9 as a melanoma metastasis gene. Cell 125 : 1269-1281, 2006.

11. Kim SH, Xia D, Dubois RN, et al: Human enhancer of filamentation 1 is a mediator of hypoxia-inducible factor- $1 \alpha$-mediated migration in colorectal carcinoma cells. Cancer Res 70: 4054-4063, 2010.

12. Thaole B, Vu HA, Yasuda K, et al: Cas-L was overexpressed in imatinib-resistant gastrointestinal stromal tumor cells. Cancer Biol Ther 8: 683-688, 2009.

13. Chang JX, Zhao GQ, Zhang GJ, et al: Expression and clinical significance of NEDD9 in lung tissues. Med Oncol 29: 2654-2660, 2012.

14. Kondo S, Iwata S, Yamada T, et al: Impact of the integrin signaling adaptor protein NEDD9 on prognosis and metastatic behavior of human lung cancer. Clin Cancer Res 18: 6326-6338, 2012.

15. Feng Y, Wang Y, Wang Z, et al: The CRTC1-NEDD9 signaling axis mediates lung cancer progression caused by LKB1 loss. Cancer Res 72: 6502-6511, 2012.

16. Miao Y, Li AL, Wang L, et al: Overexpression of NEDD9 is associated with altered expression of E-cadherin, $\beta$-catenin and $\mathrm{N}$-cadherin and predictive of poor prognosis in non-small cell lung cancer. Pathol Oncol Res 19: 281-286, 2013.

17. Sen GL and Blau HM: A brief history of RNAi: the silence of the genes. FASEB J 20: 1293-1299, 2006.
18. Chang JX, Zhao GQ, Zhang GJ, et al: Construction and characterization of a eukaryotic expression vector for small interfering RNA targeting the NEDD9 gene. Int J Mol Med 30: 1343-1348, 2012.

19. Tanabe H, Yagihashi A, Tsuji N, et al: Expression of survivin mRNA and livin mRNA in non-small-cell lung cancer. Lung Cancer 46: 299-304, 2004.

20. Sun BS, Dong QZ, Ye QH, et al: Lentiviral-mediated miRNA against osteopontin suppresses tumor growth and metastasis of human hepatocellular carcinoma. Hepatology 48: 1834-1842, 2008.

21. Singh MK, Dadke D, Nicolas E, et al: A novel Cas family member, HEPL, regulates FAK and cell spreading. Mol Biol Cell 19: 1627-1636, 2008

22. Fashena SJ, Einarson MB, Golemis EA, et al: Dissection of HEF1-dependent functions in motility and transcriptional regulation. J Cell Sci 115: 99-111, 2002.

23. Klemke RL, Leng J, Cheresh DA, et al: CAS/Crk coupling serves as a 'molecular switch' for induction of cell migration. J Cell Biol 140: 961-972, 1998.

24. Pugacheva EN and Golemis EA: The focal adhesion scaffolding protein HEF1 regulates activation of the Aurora-A and Nek2 kinases at the centrosome. Nat Cell Biol 7: 937-946, 2005.

25. Dadke D, Jarnik M, Pugacheva EN, et al: Deregulation of HEF1 impairs M-phase progression by disrupting the RhoA activation cycle. Mol Biol Cell 17: 1204-1217, 2006.

26. Seo S, Asai T, Saito T, et al: Crk-associated substrate lymphocyte type is required for lymphocyte trafficking and marginal zone B cell maintenance. J Immunol 175: 3492-3501, 2005.

27. Elbashir SM, Harborth J, Lendeckel W, et al: Duplexes of 21-nucleotide RNAs mediate RNA interference in cultured mammalian cells. Nature 411: 494-498, 2001.

28. Echeverri CJ and Perrimon N: High-throughput RNAi screening in cultured cells: a user's guide. Nat Rev Genet 7: 373-384, 2006.

29. Mofat J, Grueneberg DA, Yang X, et al: A lentiviral RNAi library for human and mouse genes applied to an arrayed viral high-content screen. Cell 124: 1283-1298, 2006. 